\title{
VOIGT PROFILE FUNCTIONS IN THE COMPLEX DOMAIN
}

\author{
A. REICHEL \\ (received 15 March 1964)
}

\section{Introduction}

The functions

$$
\begin{aligned}
\psi(x, \xi) & =\frac{\xi}{2 \sqrt{ } \pi} \int_{-\infty}^{\infty} \frac{e^{-t \xi^{2}(x-y)^{2}} d y}{1+y^{2}} \\
\phi(x, \xi) & =\frac{\xi}{2 \sqrt{ } \pi} \int_{-\infty}^{\infty} \frac{e^{-1 \xi^{2}(x-y)^{2}} y d y}{1+y^{2}}
\end{aligned}
$$

where $\xi>0$, are essentially Fourier convolutions of Gaussian and Cauchy type distributions, and have thus found application in many fields. In reactor theory, and no doubt elsewhere, the need has arisen to analyse complicated integrals and functional equations involving $\psi$ and $\phi$, the socalled Voigt profiles.

Recently Keane and Clancy [1] pointed out the advantage of extending the definition of the function

$$
\psi(x, \xi)+i \phi(x, \xi)
$$

to the complex domain. They define a function (in the present notation)

$$
\chi(z, \xi)=\psi(z, \xi)+i \phi(z, \xi)
$$

as a solution of the differential equation

$$
\frac{2}{\xi^{2}} \frac{d \chi}{d z}+(z+i) \chi=i \text {. }
$$

By developing asymptotic expansions of $\chi(z, \xi)$ in specified ranges, Keane and Clancy have evaluated a number of integrals involving $\psi(x, \xi)+i \phi(x, \xi)$ over the real axis by taking the functional of $\chi(z, \xi)$ around an infinite semicircle in the upper half-plane and using the calculus of residues. These authors also drew attention to the work of Buckler and Pull [2] who introdurced the function

$$
G(w)=\int_{0}^{\infty} \frac{e^{-u^{4}} w d u}{u^{2}+w^{2}}
$$


where $w$ is complex, and $G(w)$ is proportional to $\psi+i \phi$ when $w=\frac{1}{2} \xi(1-i x)$. There are many ways in which the extension of definition to the complex domain can be accomplished. In this paper a method which involves the use of Cauchy integrals is proposed. Although this method has features in common with both the above, it also involves a generalization of a function considered by Faddeyeva and Terent'ev [3], and leads to helpful definitions of $\psi(z, \xi)$ and $\phi(z, \xi)$ as functions of a complex variable.

The integrals discussed by Keane and Clancy are special cases of some convolution integrals which are currently important in attempts to solve problems associated with resonance overlap in reactor systems. The Cauchy integral method is used here to evaluate some of these integrals and to derive further properties of the Voigt profiles.

The function $\psi(x, \xi)$ in particular has been extensively tabulated; in two volumes by Rose et al. [4] and slightly more accurately by Bell et al. [5]. There are many short tables scattered throughout the literature of several fields; two efforts by Australians are mentioned, Posener [6], and Cook and Elliot [7], which also contains a table of $\phi(x, \xi)$. A very rapid method of evaluating the functions is given as a special case of formulae derived in the sequel.

\section{Cauchy integrals}

It can be readily shown that

$$
\begin{aligned}
& \varphi(x, \xi)=\int_{0}^{\infty} e^{-p-p^{2} / \xi^{2}} \cos p x d p \\
& \phi(x, \xi)=\int_{0}^{\infty} e^{-p-p^{2} / \xi^{2}} \sin p x d p
\end{aligned}
$$

so that

$$
\begin{aligned}
\psi(x, \xi)+i \phi(x, \xi) & =\int_{0}^{\infty} e^{-p-p^{2} / \xi^{2}} e^{i p x} d p \\
& =\lim _{y \rightarrow 1} \int_{0}^{\infty} e^{i p z} e^{-p^{2} / \xi^{2}} d p
\end{aligned}
$$

where $z=x+i y$,

$$
\begin{aligned}
& =\lim _{y \rightarrow 1} \frac{\xi}{2 \sqrt{ } \pi} \int_{-\infty}^{\infty} e^{-t \xi^{2} t^{2}} d t \int_{0}^{\infty} e^{-i p(t-z)} d p \\
& =\lim _{y \rightarrow 1} \frac{\xi}{2 \sqrt{ } \pi i} \int_{-\infty}^{+\infty} \frac{e^{-t \xi^{2} t^{2}} d t}{t-z}
\end{aligned}
$$


We therefore consider the Cauchy integral

$$
F(z)=\frac{\alpha}{\sqrt{ } \pi i} \int_{-\infty}^{+\infty} \frac{e^{-\alpha^{2} t^{2}} d t}{t-z}
$$

where $\alpha=\frac{1}{2} \xi . F(z)$ is holomorphic in the upper and lower half planes but not on the real axis, where there is a line of discontinuity. $F(z)$ is, however, continuous from the left and the right of the real axis. A formal re-arrangement of equation (7) shows that

$$
F(z)=-F(-z)=-\frac{2 \alpha}{\sqrt{ } \pi} G(i \alpha z)
$$

where $G(w)$ is the function considered by Buckler and Pull, [2], equation (3). The function considered by Faddeyeva and Terent'ev, [3], is essentially equation (7) with $\alpha=1$.

\section{Convolution integrals}

The Cauchy integral technique is first illustrated by a trivial generalization of a result given without detail by Keane and Clancy, [1], (equation (14)).

Consider a contour $C$ consisting of a rectangle of unit height, standing in the upper half plane, on the segment $-R, R$ of the real axis. When $z=x+i, F(z)=\psi(x, \xi)+i \phi(x, \xi)$.

1. Consider, for real $a$,

(9) $\oint_{C}(z-i+a)^{m}\{F(z)\}^{n} d z$

$=\left(\frac{\alpha}{\sqrt{ } \pi i}\right)^{n} \int_{-\infty}^{+\infty} \cdots \int_{-\infty}^{+\infty} e^{-\alpha^{2}\left(t_{1}^{2}+t_{2}^{2}+\cdots+t_{n}^{2}\right)} d t_{1} \cdots d t_{n} \oint_{C} \frac{(z-i+a)^{m} d z}{\left(t_{1}-z\right)\left(t_{2}-z\right) \cdots\left(t_{n}-z\right)}$

and let the contour integral be denoted by $\oint_{C} G(z) d z$.

If $m<n$, the integrals on $(0,1)$, when $z=R+i y,-R+i y$, vanish as $R \rightarrow \infty$.

If we consider the partial fractions of the proper rational function $G(z)$, we find that the residue at $z=t_{k}$ say, is $(-1)$ times

$$
\frac{\left(t_{k}-i+a\right)^{m}}{\left(t_{1}-t_{k}\right)\left(t_{2}-t_{k}\right) \cdots\left(t_{k-1}-t_{k}\right)\left(t_{k+1}-t_{k}\right) \cdots\left(t_{n}-t_{k}\right)} .
$$

If $m<n-1$, this rational function in $t_{k}$ is proper and its partial fractions in $t_{k}$ will reproduce all the other residues with opposite sign.

If $m=n-1$, the function can be rearranged as 


$$
(-)^{n-1}+\frac{\left(t_{k}-i+a\right)^{m}+(-1)^{m+1}\left(t_{1}-t_{k}\right) \cdots\left(t_{k-1}-t_{k}\right)\left(t_{k+1}-t_{k}\right) \cdots\left(t_{n}-t_{k}\right)}{\left(t_{1}-t_{k}\right) \cdots\left(t_{k-1}-t_{k}\right)\left(t_{k+1}-t_{k}\right) \cdots\left(t_{n}-t_{k}\right)} .
$$

The new rational function is proper in $t_{k}$ and its partial fractions in $t_{k}$ will cancel all other residues as before.

Thus

$$
\oint_{C} G(z) d z=\left\{\begin{array}{lll}
-\pi i(-1)^{n-1} & \text { if } & m=n-1 \\
=0, & \text { if } & m<n-1 .
\end{array}\right.
$$

In addition, the integral on the real axis, from $-\infty$ to $+\infty$ vanishes in the sense of Cauchy principal value, and so, finally,

$$
\int_{-\infty}^{+\infty}(x+a)^{m}[\psi(x, \xi)+i \phi(x, \xi)]^{n} d x=\left\{\begin{array}{lll}
\pi i^{n-1} & \text { for } & m=n-1 \\
0 & \text { for } & m<n-1
\end{array}\right.
$$

Similarly, by considering $\oint_{C}\{F(z)\}^{n} /(z-i+a)^{m} d z, m=1,2, \cdots$ we find

$$
\int_{-\infty}^{+\infty} \frac{[\psi(x, \xi)+i \phi(x, \xi)]^{n}}{(x+a)^{m}} d x=\frac{(-1)^{m-1} \pi i}{(m-1) !} \frac{d^{m-1}}{d a^{m-1}}\left\{[\psi(a, \xi)-i \phi(a, \xi)]^{n}\right\}
$$

2. The extension of the present method to include integrals of $F(z)$ or powers of $F(z)$ multiplied by functions with poles either inside or outside $C$ is obvious. The most striking of these are the following.

Consider, for real $a$,

$$
\begin{aligned}
\oint_{C} F(z) F(a-z+2 i) d z & \\
= & \left(\frac{\alpha}{\sqrt{ } \pi i}\right)^{2} \int_{-\infty}^{+\infty} \int_{-\infty}^{+\infty} e^{-\alpha^{8}\left(t_{1}^{2}+t_{2}^{2}\right)} d t_{1} d t_{2} \oint_{C} \frac{d z}{\left(t_{1}-z\right)\left[t_{2}-(a-z+2 i)\right]} .
\end{aligned}
$$

Now

$$
\oint_{C} \frac{d z}{\left(t_{1}-z\right)\left[t_{2}-(a-z+2 i)\right]}=\frac{-\pi i}{t_{1}+t_{2}-a-2 i}
$$

Also,

Hence

$$
\lim _{R \rightarrow \infty} \int_{-R}^{+R} \frac{d x}{\left(t_{1}-x\right)\left(x+t_{2}-a-2 i\right)}=\frac{\pi i}{t_{1}+t_{2}-a-2 i}
$$

$$
\begin{aligned}
I & =\int_{-\infty}^{+\infty}[\psi(x, \xi)+i \phi(x, \xi)][\psi(a-x, \xi)+i \phi(a-x, \xi)] d x \\
& =2 \pi i\left(\frac{\alpha}{\sqrt{ } \pi i}\right)^{2} \int_{-\infty}^{+\infty} \int_{-\infty}^{+\infty} \frac{e^{-\alpha^{2}\left(t_{1}^{2}+t_{2}^{2}\right)} d t_{1} d t_{2}}{\left(t_{1}+t_{2}-a-2 i\right)} .
\end{aligned}
$$

Let $u+v=t_{1}, u-v=t_{2}$, and so 


$$
\begin{aligned}
I & =2 \pi i\left(\frac{\alpha}{\sqrt{ } \pi i}\right)^{2} \int_{-\infty}^{+\infty} e^{-2 \alpha^{2} v^{2}} d v \int_{-\infty}^{+\infty} \frac{e^{-2 \alpha^{2} u^{2}} d u}{u-\left(\frac{1}{2} a+i\right)} \\
& =\pi\left[\psi\left(\frac{1}{2} a, \sqrt{ } 2 \xi\right)+i \phi\left(\frac{1}{2} a, \sqrt{ } 2 \xi\right)\right]
\end{aligned}
$$

Consideration of the special case $a=0$, together with the fact that, from equation (10) when $n=2, m=0$,

$$
\int_{-\infty}^{+\infty} \psi^{2} d x=\int_{-\infty}^{+\infty} \phi^{2} d x, \text { etc. }
$$

suggests

and

$$
\begin{aligned}
& \int_{-\infty}^{+\infty} \psi(x, \xi) \psi(a-x, \xi) d x=\frac{1}{2} \pi \psi\left(\frac{1}{2} a, \sqrt{ } 2 \xi\right) \\
& \int_{-\infty}^{+\infty} \phi(x, \xi) \phi(a-x, \xi) d x=-\frac{1}{2} \pi \psi\left(\frac{1}{2} a, \sqrt{ } 2 \xi\right)
\end{aligned}
$$

$$
\int_{-\infty}^{+\infty} \psi(x, \xi) \phi(a-x, \xi) d x=\frac{1}{2} \pi \phi\left(\frac{1}{2} a, \sqrt{ } 2 \xi\right)
$$

We thus have an interesting group-like property. This result can be easily generalized to the four parameter situation in reactor theory when resonances of two nuclear species overlap due to Doppler broadening. There is an analogue in spectral analysis. We have

$$
\begin{aligned}
\int_{-\infty}^{+\infty}\left[\psi\left(x, \xi_{1}\right)+i \phi\left(x, \xi_{1}\right)\right]\left[\psi\left(\frac{x^{\prime}-x}{K}, \xi_{2}\right)+i \phi\left(\frac{x^{\prime}-x}{K}, \xi_{2}\right)\right] d x \\
=\frac{2 K \pi}{K+1}\left[\psi\left(\frac{x^{\prime}}{K+1}, \frac{(K+1) \xi_{1} \xi_{2}}{\left(K^{2} \xi_{1}^{2}+\xi_{2}^{2}\right)^{\frac{1}{2}}}\right)+i \phi\left(\frac{x^{\prime}}{K+1}, \frac{(K+1) \xi_{1} \xi_{2}}{\left(K^{2} \xi_{1}^{2}+\xi_{2}^{2}\right)^{\frac{1}{t}}}\right)\right] .
\end{aligned}
$$

In the above, $x^{\prime}$ is a measure of separation of resonance centres and $K$ is a ratio of resonance widths.

Thus

$$
\begin{aligned}
& \int_{-\infty}^{+\infty} \psi\left(x, \xi_{1}\right) \psi\left(\frac{x^{\prime}-x}{K}, \xi_{2}\right) d x=\frac{K \pi}{K+1} \psi\left(\frac{x^{\prime}}{K+1}, \frac{(K+1) \xi_{1} \xi_{2}}{\left(K^{2} \xi_{1}^{2}+\xi_{2}^{2}\right)^{\frac{1}{2}}}\right) \\
& \int_{-\infty}^{+\infty} \phi\left(x, \xi_{1}\right) \phi\left(\frac{x^{\prime}-x}{K}, \xi_{2}\right) d x=\frac{-K \pi}{K+1} \psi\left(\frac{x^{\prime}}{K+1}, \frac{(K+1) \xi_{1} \xi_{2}}{\left(K^{2} \xi_{1}^{2}+\xi_{2}^{2}\right)^{\frac{1}{t}}}\right) \\
& \int_{-\infty}^{+\infty} \psi\left(x, \xi_{1}\right) \phi\left(\frac{x^{\prime}-x}{K}, \xi_{2}\right) d x=\frac{K \pi}{K+1} \phi\left(\frac{x^{\prime}}{K+1}, \frac{(K+1) \xi_{1} \xi_{2}}{\left(K^{2} \xi_{1}^{2}+\xi_{2}^{2}\right)^{\frac{1}{2}}}\right) .
\end{aligned}
$$


When $\xi \rightarrow \infty$,

and

$$
\psi\left(\frac{x}{K}, \xi\right) \rightarrow \frac{K^{2}}{K^{2}+x^{2}}
$$

$$
\phi\left(\frac{x}{K}, \xi\right) \rightarrow \frac{K x}{K^{2}+x^{2}} .
$$

These are the "natural" profiles. The above equations then give a variety of convolution formulae, e.g.

$$
\int_{-\infty}^{+\infty} \frac{\psi\left(x, \xi_{1}\right) d x}{K^{2}+\left(x-x^{\prime}\right)^{2}}=\frac{\pi}{K(K+1)} \psi\left(\frac{x^{\prime}}{K+1},(K+1) \xi_{1}\right)
$$

and so on.

Many others may be obtained by differentiation under the integral sign with respect to the parameters $x^{\prime}$ or $K$, and using the differential equations

$$
\begin{aligned}
& x \psi=\phi-\frac{2}{\xi^{2}} \frac{\partial \psi}{\partial x} \\
& x \phi=1-\psi-\frac{2}{\xi^{2}} \frac{\partial \phi}{\partial x} .
\end{aligned}
$$

Convolutions with Gaussians may also be obtained by considering

We find

$$
\oint_{C} e^{-\alpha_{1}^{2}(z-i)^{2}} F\left(x^{\prime}-z+2 i\right) d z
$$

$$
\begin{aligned}
\int_{-\infty}^{+\infty} e^{-t \xi_{1}^{\prime}}\left[\psi\left(x^{\prime}-x, \xi_{2}\right)+i \phi\left(x^{\prime}-x, \xi_{2}\right)\right] d x \\
=\frac{2 \sqrt{\pi}}{\xi_{1}}\left[\psi\left(x^{\prime}, \frac{\xi_{1} \xi_{2}}{\left(\xi_{1}^{2}+\xi_{2}^{2}\right)^{\frac{1}{2}}}\right)+i \phi\left(x^{\prime}, \frac{\xi_{1} \xi_{2}}{\left(\xi_{1}^{2}+\xi_{2}^{2}\right)}\right)\right]
\end{aligned}
$$

which may be decomposed as before.

\section{Series expansions}

For

$$
F(z)=\frac{\alpha}{\sqrt{ } \pi i} \int_{-\infty}^{+\infty} \frac{e^{-\alpha^{2} t^{2}} d t}{t-z}
$$

we have, on differentiating under the integral sign and integrating by parts,

$$
F^{\prime}(z)+2 \alpha^{2} z F(z)=2 \alpha^{2} i \text {. }
$$


The special case $\alpha=1$ has been considered essentially by Faddeyeva and Terent'ev [3].

From equation (5) we find

$$
F(0)=\alpha \sqrt{ } \pi,
$$

if $z \rightarrow 0$ from the upper half-plane.

Also, by successive differentiation of equation (24),

$$
F^{(n)}(z)+2 \alpha^{2} z F^{(n-1)}(z)+(n-1) 2 \alpha^{2} F^{(n-2)} z=0, \quad n \geqq 2 .
$$

Towards obtaining a Maclaurin expansion, we have

and

$$
F(0)=\alpha \sqrt{ } \pi, \quad F^{\prime}(0)=2 \alpha^{2} i,
$$

$$
\begin{aligned}
F^{(2 m)}(0) & =(-2)^{m}(2 m-1)(2 m-3) \cdots 1 \cdot\left(\alpha^{2}\right)^{m} F(0) \\
F^{(2 m+1)}(0) & =(-2)^{m}(2 m)(2 m-2)(2 m-4) \cdots 2 \cdot\left(\alpha^{2}\right)^{m} F^{\prime}(0) .
\end{aligned}
$$

The coefficient of $z^{2 m}$ in the Maclaurin expansion is $\left(i^{2 m} \alpha^{2 m+1} \sqrt{ } \pi\right) / m$ ! and the coefficient of $z^{2 m+1}$ is $\left(i^{2 m+1} \alpha^{2 m+2} \sqrt{ } \pi\right) /\left(m+\frac{1}{2}\right) !$. Thus the coefficient of $z^{n}$ is $\left(i^{n} \alpha^{n+1} \sqrt{ } \pi\right) /\left(\frac{1}{2} n\right) !$ and so

and

$$
F(z)=\sqrt{ } \pi \alpha \sum_{n=0}^{\infty} \frac{(i \alpha z)^{n}}{\left(\frac{1}{2} n\right) !}
$$

$$
\psi(x, \xi)+i \phi(x, \xi)=\frac{1}{2} \xi \sqrt{ } \pi \sum_{n=0}^{\infty} \frac{[i \xi(x+i)]^{n}}{2^{n}\left(\frac{1}{2} n\right) !} .
$$

Towards obtaining an asymptotic development we find

$$
\begin{aligned}
F(z) & =-\frac{\alpha}{\sqrt{ } \pi i} \int_{-\infty}^{+\infty} \frac{e^{-\alpha^{2} t^{2}} d t}{z-t} \\
& =-\frac{\alpha}{\sqrt{ } \pi i} \int_{-\infty}^{+\infty} e^{-\alpha^{2} t^{2}}\left[\frac{1}{z}+\frac{t}{z^{2}}+\cdots+\frac{t^{2 k}}{z^{2 k+1}}+\frac{t^{2 k+1}}{z^{2 k+2}}+\cdots\right] d t .
\end{aligned}
$$

Only even powers of $t$ are relevant and we find

$$
\frac{\alpha}{\sqrt{ } \pi i} \int_{-\infty}^{+\infty} \frac{e^{-\alpha^{2} t^{2}} d t}{t-z}=F(z)=-\frac{\alpha}{\sqrt{ } \pi i} \sum_{k=0}^{n} \frac{\Gamma\left(k+\frac{1}{2}\right)}{(\alpha z)^{2 k+1}}+R_{n} .
$$

This result has also been given by Buckler and Pull [2], in view of equation (3) and equation (8). Putting $z=x+i$ and separating real and imaginary parts we obtain the well-known asymptotic developments of $\psi(x, \xi)$ and $\phi(x, \xi)$. In particular, as $\xi \rightarrow \infty$,

$$
\psi(x, \xi) \rightarrow \frac{1}{1+x^{2}} \text { and } \phi(x, \xi) \rightarrow \frac{x}{1+x^{2}} .
$$




\section{Definitions of $\psi(z, \xi)$ and $\phi(z, \xi)$}

A simple mapping gives

where

$$
\psi(x, \xi)+i \phi(x, \xi)=\lim _{\nu \rightarrow 0} W(z)
$$

$$
W(z)=\frac{\alpha}{\sqrt{ } \pi i} \int_{-\infty}^{+\infty} \frac{e^{-\alpha^{2} t^{2}} d t}{t-(z+i)}=u(x, y)+i v(x, y)
$$

$W(z)$ has a line of discontinuity at $y=-1$. For $y>-1$, we have, on separating real and imaginary parts,

$$
\begin{aligned}
& u(x, y)=\frac{\alpha}{\sqrt{ } \pi} \int_{-\infty}^{+\infty} \frac{e^{-\alpha^{2} t^{2}}(y+1) d t}{(x-t)^{2}+(y+1)^{2}} \\
& v(x, y)=\frac{\alpha}{\sqrt{ } \pi} \int_{-\infty}^{+\infty} \frac{(x-t) e^{-\alpha^{2} t^{2}} d t}{(x-t)^{2}+(y+1)^{2}} .
\end{aligned}
$$

Clearly, on $y=0$, we recover the Voigt profiles. The functions $u(x, y)$ and $v(x, y)$ have been tabulated by Faddeyeva and Terent'ev [3] in the special case $\alpha=1, \sqrt{ } \pi$ replaced by $\pi$ and $(y+1)$ replaced by $y$. This would seem to be an unnecessary tabulation since a simple change of variable gives

so that for $y>-1$

$$
\begin{aligned}
& u(x, y)=\frac{1}{y+1} \psi\left(\frac{x}{1+y}, \xi(y+1)\right) \\
& v(x, y)=\frac{1}{y+1} \phi\left(\frac{x}{1+y}, \xi(y+1)\right)
\end{aligned}
$$

$$
W(z)=\frac{1}{y+1}\left\{\psi\left(\frac{x}{1+y}, \xi(1+y)\right)+i \phi\left(\frac{x}{1+y}, \xi(1+y)\right)\right\} .
$$

Let $W^{+}(x)$ denote the value of $W(z)$ as $y \rightarrow-1$ from the upper half-plane, and $W-(x)$ the value of $W(z)$ as $y \rightarrow-1$ from the lower half-plane. Then, applying the Plemelj formulae for Cauchy integrals on the line of integration we have

$$
\begin{aligned}
& W^{+}(x)=\frac{1}{2} \cdot \frac{2 \pi \alpha}{\sqrt{ } \pi} e^{-\alpha^{2} x^{2}}+\frac{1}{2 \pi i} \frac{2 \pi \alpha}{\sqrt{ } \pi} \int_{-\infty}^{+\infty} \frac{e^{-\alpha^{2} t^{2}} d t}{t-x} \\
& W^{-}(x)=-\frac{1}{2} \cdot \frac{2 \pi \alpha}{\sqrt{ } \pi} e^{-\alpha^{2} x^{2}}+\frac{2 \pi \alpha}{\sqrt{ } \pi 2 \pi i} \int_{-\infty}^{+\infty} \frac{e^{-\alpha^{2} t^{2}} d t}{t-x} .
\end{aligned}
$$

The integral is easily evaluated by the substitution $u=t-x$ and differentiation under the integral sign to form a differential equation, and finally 


$$
\begin{aligned}
& W+(x)=\sqrt{ } \pi \alpha e^{-\alpha^{2} x^{2}}+2 i \alpha e^{-\alpha^{2} x^{2}} \int_{0}^{\alpha x} e^{t^{2}} d t \\
& W-(x)=-\sqrt{ } \pi \alpha e^{-\alpha^{2} x^{2}}+2 i \alpha e^{-\alpha^{2} x^{2}} \int_{0}^{\alpha x} e^{t^{2}} d t .
\end{aligned}
$$

The integral $\int_{0}^{\alpha x} e^{t^{2}} d t$ has been tabulated by Dawson [10] and Terrill and Sweeney [11].

Hence, a solution of the Hilbert problem

$$
W^{+}(x)-W^{-}(x)=2 \sqrt{ } \pi \alpha e^{-\alpha^{2} x^{2}} \text { on } y=-1
$$

is $W(z)$. It can readily be shown to be the only solution.

On the imaginary axis, $W(z)$ is real. From equations $(34),(35),(36)$,

$$
\begin{aligned}
& W(i y)=\frac{1}{y+1} \psi(0, \xi(1+y)) \\
& W+(0)=\frac{\sqrt{ } \pi \xi}{2} \\
& W-(0)=-\frac{\sqrt{ } \pi \xi}{2}
\end{aligned}
$$

Equation (34) shows that $W(z)$ takes the same values as Keane and Clancy's, [1], function $\chi(z)$ when $y>-1$, but whereas $\chi(z)$ is an integral function, continuous across $y=-1$ with an isolated essential singularity at infinity, $W(z)$ is a sectionally holomorphic function.

Since

we have

$$
\begin{aligned}
\psi+i \phi & =\frac{\alpha}{\sqrt{ } \pi i} \int_{-\infty}^{+\infty} \frac{e^{-\alpha^{2} t^{2}} d t}{t-(x+i)} \\
\psi-i \phi & =\frac{\alpha}{\sqrt{ } \pi i} \int_{-\infty}^{+\infty} \frac{e^{-\alpha^{2} t^{2}} d t}{t+x-i}
\end{aligned}
$$

$$
\begin{aligned}
& \psi(x, \xi)=\frac{\alpha}{\sqrt{ } \pi i} \int_{-\infty}^{+\infty} \frac{e^{-\alpha^{2} t^{2}}(t-i) d t}{(t-i)^{2}-x^{2}} \\
& \phi(x, \xi)=-\frac{\alpha x}{\sqrt{ } \pi} \int_{-\infty}^{+\infty} \frac{e^{-\alpha^{2} t^{2}} d t}{(t-i)^{2}-x^{2}}
\end{aligned}
$$

Equations (39) and (40) give asymptotic expansions in a form convenient for computation, viz.:

$$
\begin{aligned}
\psi(x, \xi)=x^{-2}+\left(6 \xi^{-2}-1\right) x^{-4} & +\left(60 \xi^{-4}-20 \xi^{-2}+1\right) x^{-6} \\
& +\left(840 \xi^{-6}-420 \xi^{-4}+42 \xi^{-2}-1\right) x^{-8}+\cdots
\end{aligned}
$$




$$
\begin{aligned}
\phi(x, \xi)=x^{-1}+\left(2 \xi^{-2}-1\right) x^{-3} & +\left(12 \xi^{-4}-12 \xi^{-2}+1\right) x^{-5} \\
& +\left(120 \xi^{-6}-180 \xi^{-4}+30 \xi^{-2}-1\right) x^{-7}+\cdots .
\end{aligned}
$$

We define

where $z=x+i y$.

$$
\begin{aligned}
& \psi(z, \xi)=\frac{\alpha}{\sqrt{ } \pi i} \int_{-\infty}^{+\infty} \frac{e^{-\alpha^{2} t^{2}}(t-i) d t}{(t-i)^{2}-z^{2}} \\
& \phi(z, \xi)=-\frac{\alpha z}{\sqrt{ } \pi} \int_{-\infty}^{+\infty} \frac{e^{-\alpha^{2} t^{2}} d t}{(t-i)^{2}-z^{2}}
\end{aligned}
$$

Clearly

$$
\begin{aligned}
& \psi(z, \xi)=\psi(-z, \xi) \\
& \phi(z, \xi)=-\phi(-z, \xi) .
\end{aligned}
$$

Many of the convolution integrals follow simply from equations (43) and (44) and the calculus of residues. For example, the Hilbert transform property (Keane and Clancy, [1]) is readily proved by taking

$$
\oint_{C_{1}} \frac{\psi(z, \xi)}{z-a} d z
$$

where $C_{1}$ is an infinite semi-circle in the upper half-plane. Since

$$
\oint_{c_{1}} \frac{d z}{(z-a)\left[(t-i)^{2}-z^{2}\right]}=\frac{\pi i}{(t-i)^{2}-a^{2}}+\frac{2 \pi i}{2(-t+i-a)(t-i)}
$$

we have

$$
\begin{aligned}
\int_{-\infty}^{+\infty} \frac{\psi(x, \xi)}{x-a} d x & =\frac{\pi i \alpha}{\sqrt{ } \pi i} \int_{-\infty}^{+\infty} \frac{e^{-\alpha^{2} t^{2}}(t-i) d t}{(t-i)^{2}-a^{2}}-\frac{2 \pi i \alpha}{2 \sqrt{ } \pi i} \int_{-\infty}^{+\infty} \frac{e^{-\alpha^{2} t^{2}} d t}{t-(-a+i)} \\
& =\pi i \varphi(a, \xi)-\pi i[\psi(a, \xi)-i \phi(a, \xi)] \\
& =-\pi \phi(a, \xi) .
\end{aligned}
$$

Both $\psi(z, \xi)$ and $\phi(z, \xi)$ are analytic within and outside the strip $-1<y<+1$ but are discontinuous across $y=-1, y=+1$. The values of $\psi(z, \xi)$ and $\phi(z, \xi)$ are readily given in terms of the values of $\psi$ and $\phi$ on the real axis. In fact,

$$
\begin{aligned}
\varphi(z, \xi) & =\frac{1}{2(1+y)} \psi\left(\frac{x}{1+y}, \xi(1+y)\right)+\frac{i}{2(1+y)} \phi\left(\frac{x}{1+y}, \xi(1+y)\right) \\
& +\frac{1}{2(1-y)} \psi\left(\frac{x}{1-y}, \xi(1-y)\right)-\frac{i}{2(1-y)} \phi\left(\frac{x}{1-y}, \xi(1-y)\right)
\end{aligned}
$$

for $-1<y<+1$; and for $y>+1$ 


$$
\begin{aligned}
\psi(z, \xi) & =\frac{1}{2(1+y)} \psi\left(\frac{x}{1+y}, \xi(1+y)\right)+\frac{i}{2(1+y)} \phi\left(\frac{x}{1+y}, \xi(1+y)\right) \\
& -\frac{1}{2(y-1)} \psi\left(\frac{x}{y-1}, \xi(y-1)\right)-\frac{i}{2(y-1)} \phi\left(\frac{x}{y-1}, \xi(y-1)\right) .
\end{aligned}
$$

Clearly the values on the imaginary axis are real and conjugate complex values are taken on either side of the imaginary axis. This, together with equation (45) gives the values everywhere from those in the first quadrant.

Similarly,

$$
\begin{aligned}
\phi(z, \xi) & =\frac{1}{2 i(1+y)} \psi\left(\frac{x}{1+y}, \xi(1+y)\right)+\frac{1}{2(1+y)} \phi\left(\frac{x}{1+y}, \xi(1+y)\right) \\
& -\frac{1}{2 i(1-y)} \psi\left(\frac{x}{1-y}, \xi(1-y)\right)+\frac{1}{2(1-y)} \phi\left(\frac{x}{1-y}, \xi(1-y)\right)
\end{aligned}
$$

for $-1<y<+1$; and for $y>+1$,

$$
\begin{aligned}
\phi(z, \xi) & =\frac{1}{2 i(y+1)} \psi\left(\frac{x}{y+1}, \xi(y+1)\right)+\frac{1}{2(y+1)} \phi\left(\frac{x}{y+1}, \xi(y+1)\right) \\
& +\frac{1}{2 i(y-1)} \psi\left(\frac{x}{y-1}, \xi(y-1)\right)+\frac{1}{2(y-1)} \phi\left(\frac{x}{y-1}, \xi(y-1)\right) .
\end{aligned}
$$

On the imaginary axis, $\phi(z, \xi)$ is pure imaginary.

\section{Expansions in Hermite polynomials and computation}

Using the integral formula for the Hermite Polynomials, viz.

$$
H_{m}(x)=\frac{2^{m}}{\sqrt{ } \pi} \int_{-\infty}^{+\infty}(x+i t)^{m} e^{-t^{2}} d t
$$

we have, for real $a$,

$$
\begin{aligned}
I_{m}(a) & \left.=\int_{-\infty}^{+\infty} e^{-x^{2}} H_{m}(x)\{\psi(a-x), \xi)+i \phi(a-x, \xi)\right\} d x \\
& =\frac{\alpha}{\sqrt{ } \pi i} \frac{2^{m}}{\sqrt{ } \pi} \int_{-\infty}^{+\infty} e^{-v^{2}} d v \int_{-\infty}^{+\infty} e^{-a^{2} t^{2}} d t \int_{-\infty}^{+\infty} \frac{e^{-x^{2}}(x+i v)^{m} d x}{x+t-(a+i)} \\
& =\frac{\alpha}{\sqrt{ } \pi i} \frac{2^{m}}{\sqrt{ } \pi} K^{m+2} \int_{-\infty}^{+\infty} \int_{-\infty}^{+\infty} e^{-K^{2} v^{2}-\alpha^{2} K s^{2}} d v d s \int_{-\infty}^{+\infty} \frac{e^{-K r^{2}}(r-\beta)^{m} d r}{r-(a+i)}
\end{aligned}
$$

where

and

$$
\beta=s-i v
$$




$$
K=\frac{\alpha^{2}}{1+\alpha^{2}}=\frac{\xi^{2}}{4+\xi^{2}}
$$

It is easily shown that

$$
\begin{aligned}
& I_{0}(a)=\sqrt{ } \pi\left[\psi\left(a, 2 K^{\frac{1}{b}}\right)+i \phi\left(a, 2 K^{\frac{1}{b}}\right)\right] \\
& I_{1}(a)=-\sqrt{ } \pi \cdot i 2 K+\sqrt{ } \pi \cdot 2 K(a+i)\left[\psi\left(a, 2 K^{\frac{1}{2}}\right)+i \phi\left(a, 2 K^{\frac{1}{2}}\right)\right] .
\end{aligned}
$$

A simple recurrence relation can be obtalned by noting that the integral

$$
\begin{aligned}
B_{n} & =\frac{\alpha}{\sqrt{ } \pi i} \frac{2^{n}}{\sqrt{ } \pi} K^{n+2} \int_{-\infty}^{+\infty} \int_{-\infty}^{+\infty} e^{-K^{2} v^{2}-\alpha^{2} K s^{2}} d v d s \int_{-\infty}^{+\infty} \frac{e^{-K r^{2}}(r-\beta)^{n} \beta d r}{r-(a+i)} \\
& =n I_{n-1} ; \quad B_{0}=0
\end{aligned}
$$

and that the integral

$$
\begin{aligned}
\rho_{n} & =\int_{-\infty}^{+\infty} \int_{-\infty}^{+\infty} \int_{-\infty}^{+\infty} e^{-K^{2} v^{2}-\alpha^{1} K_{s^{2}}-K_{r^{2}}}(\gamma-\beta)^{n} d v d s d r \\
& = \begin{cases}0, & n \neq 0 \\
\frac{\pi !}{\alpha K^{2}}, & n=0\end{cases}
\end{aligned}
$$

We obtain finally,

(58) $\quad I_{m}(a)=(a+i) 2 K I_{m-1}-2 K(m-1) I_{m-2}$, where $m=2,3, \cdots$.

Equations (54), (55), (58), together with the normalization integral

$$
\int_{-\infty}^{\infty} e^{-z^{1}} H_{m}(z) H_{n}(z) d z=\delta_{m n} 2^{n} \cdot n ! \sqrt{ } \pi
$$

give expansions of the functions $\psi(a-x, \xi)$ and $\phi(a-x, \xi)$ in Hermite polynomials. The special case $a=0$ gives an expansion of $\psi(x, \xi)$ in even order Hermite polynomials and $\phi(x, \xi)$ in odd order. We have then a very rapid method for computing $\psi(x, \xi)$ and $\phi(x, \xi)$. These expansions have been used, together with equations (41), (42) when $x>7 / \xi$.

\section{References}

[1] Keane, A. and Clancy, B. E., This Journal, 4 (1964).

[2] Buckler, Mrs A. and Pull, I., A.A.E.W. - R 226, U.K. Atomic Energy Authority. H.M.S.O. (1962).

[3] Faddeyeva, V. N. and Terent'ev, N. M., Tables of the Probability Integral for Complex Argument, Pergamon Press, (1961).

[4] Rose, M. E., Miranker, W., Leak, P., Rosenthal, L. and Hendrickson, J. K., WAPD SR - 506, Oak Ridge Technical Information Extension (1954). 
[5] Bell, V. J., Buckler, Mrs P. A. C. and Pull, I. C., A.E.E.W. - R 266, U.K. Atomic Energy Authority, H.M.S.O. (1963).

[6] Posener, D., Australian J. Phys. 12 (1959), 184.

[7] Cook, J. L. and Elliott, D., Australian J. of Applied Science, 11, No. 1 (1960), 16-32.

[8] Dawson, H. G., Proc. London Math. Soc., (1) 29 (1898), 519-522.

[9] Terrill, H. M. and Sweeney, L., Journal of Franklin Institute, 237 (1944), 495-497; 238 (1944), 220-222.

Department of Applied Mathematics,

University of Sydney. 